\title{
结合非局部低秩先验的图像超分辨重建概率模型
}

\author{
张中兴 ${ }^{1,2)}$, 刘慧 ${ }^{1,2)^{*}}$, 郭强 ${ }^{1,2)}$, 林毓秀 1,2$)$ \\ ${ }^{1)}$ (山东财经大学计算机科学与技术学院 济南 250014) \\ ${ }^{2)}$ (山东省数字媒体技术重点实验室 济南 250014) \\ (liuh_lh@sdufe.edu.cn)
}

\begin{abstract}
摘 要: 现今图像成像技术日益普及, 但受成像设备、成像环境以及在获取图像过程中外界噪声等因素的相互制约, 在实际应用中很多图像成像分辨率较低，带来诸多问题. 为此，提出一种有效的基于最大后验概率和非局部低秩先 验的图像超分辨重建模型. 首先, 该模型采用连续图像序列作为数据输人, 利用单幅图像内与连续图像间的相似性 作为先验知识, 提升相似图像块匹配度, 消除图像细节丢失现象. 然后, 以最大后验概率框架建模, 使用高斯分布和 吉布斯分布拟合模型参数, 提升模型泛化能力. 通过相似块的奇异值估计待求块的奇异值, 采用低秩截断抑制重建 过程中引人的噪声. 最后, 利用图像的非局部自相似性和低秩性质, 以非局部低秩约束正则化图像重建过程, 添加图 像的局部和全局信息来提升重建效果. 在标准光流数据集、纽约大学和山东省千佛山医院提供的数据集上的实验结 果表明，文中基于最大后验和非局部低秩先验的模型与传统插值算法、基于重建的优秀算法相比，在 5 组仿真实验中, 其平均峰值信噪比提升 $6.3 \mathrm{~dB}$, 在保持图像纹理特征和恢复图像细节方面可取得更好的重建性能.
\end{abstract}

关键词：图像超分辨重建; 最大后验概率; 非局部低秩正则化; 交替最小化

中图法分类号: TP391.41 DOI: 10.3724/SP.J.1089.2021.18389

\section{Super-Resolution Reconstruction Using Probability Model Combined with Nonlocal Low-Rank Prior}

\author{
Zhang Zhongxing ${ }^{1,2)}$, Liu Hui ${ }^{1,2)^{*}}$, Guo Qiang ${ }^{1,2)}$, and Lin Yuxiu ${ }^{1,2)}$ \\ 1) (School of Computer Science and Technology, Shandong University of Finance and Economics, Jinan 250014) \\ ${ }^{2)}$ (Digital Media Technology Key Laboratory of Shandong Province, Jinan 250014)
}

\begin{abstract}
Nowadays imaging technologies have become more and more popular. However, due to the mutual constraints from imaging equipment, environment and other factors, such as external noise in the process of acquiring images, the image resolution is generally low in the practical applications, which causes many problems. In this paper, we propose an effective image super-resolution reconstruction model based on maximum a posterior probability (MAP) and nonlocal low-rank prior (NLP). Firstly, by inputting the continuous image sequence, the similarity inside the single image and among the image sequence is used as prior knowledge, in order to improve the matching degree of similar image patches and eliminate the loss of image details. Then, the reconstruction is modeled with MAP framework, where the parameters are fitted by Gaussian distribution and Gibbs distribution, respectively, for increasing the generalization capability. Furthermore, this model estimates the singular values of the desired patches by singular values of similar patches, and suppresses the noise by low-rank
\end{abstract}

收稿日期: 2020-05-15; 修回日期: 2020-08-12. 基金项目：国家自然科学基金重点项目浙江联合基金(U1609218); 国家自然科学 基金(61572286, 61873145); 山东省重点研发计划(2017CXGC1504); 山东省省属高校优秀青年人才联合基金(ZR2017JL029); 山东省 高等学校优势学科人才团队培养计划. 张中兴(1996一), 男, 硕士研究生, 主要研究方向为图像处理、数据挖掘; 刘慧(1978一), 女, 博士, 教授, 硕士生导师, CCF 会员, 论文通讯作者, 主要研究方向为图像处理、机器学习; 郭强(1979一), 男, 博士, 教授, 硕士生导 师, CCF 会员, 主要研究方向为图像处理、计算机视觉; 林毓秀(1996一), 女, 硕士研究生, 主要研究方向为医学图像处理. 
truncation. Finally, to exploit the nonlocal self-similarity and low-rank nature of images, NLP regularization is adopted to regularize the reconstruction process, which introduces the local and global image information to improve the reconstruction effect. The experimental results on the standard optical flow datasets and the datasets provided by New York University and Shandong Provincial Qianfoshan Hospital show that, this proposed model based on MAP and NLP is comparable to the traditional interpolation algorithms and the excellent reconstruction-based algorithms. This method increases the average peak signal-to-noise ratio by $6.3 \mathrm{~dB}$ in five simulation experiments and achieves better reconstruction performance in maintaining image texture features and restoring image details.

Key words: image super-resolution reconstruction; maximum a posterior probability; nonlocal low-rank regularization; alternative minimization

视觉是人类从外界获取信息的主要途径之一, 而大部分基于视觉的应用性能依赖于图像的质量. 高分辨率(high resolution, HR)图像分辨率高, 包含 丰富的图像细节和更多的图像信息, 因此在医学 领域、视频监控领域、遥感领域等实际应用中具有 重要价值. 尽管在这些实际应用领域中，图像成像 技术已经趋于成熟，但受成像设备、成像环境和人 为干扰等因素的相互制约, 大部分图像的分辨率 很低. 例如, 在医学领域, 医学图像成像技术受医 学成像设备、放射性元素危害度以及人体生理健康 等因素的相互制约, 许多医学图像成像分辨率很 低，低分辨率(low resolution，LR)图像无法有效地 辅助病灶组织分类检测任务; 因此，图像超分辨技 术应运而生. 超分辨率(super resolution, SR)技术 是一种图像分析和处理技术, 将观测到的 LR 单幅 图像或图像序列作为输人, 生成 HR 的单幅图像或 图像序列. 基于插值的算法、基于学习的算法和基 于重建的算法是目前流行的 3 类图像 SR 算法.

(1) 基于插值的算法是一种较早提出且相对 简单的算法. 首先计算 LR 图像与目标 HR 图像之 间的配准关系, 再根据插值公式, 利用邻域内已知 像素值得到待插值点像素值，从而得到目标 HR 图 像. 常见的有双线性插值 ${ }^{[1]}$ 、双三次插值(bicubic interpolation, Bicubic $)^{[2]}$ 和最邻近插值 (nearest neighbor interpolation, $\mathrm{NNI})^{[3]}$. 基于插值的算法实 现简单、计算复杂度低，因此具有很好的实时性. 但在插值过程中未考虑图像的各向异性, 不能有 效地保留图像高频信息，导致放大后图像的轮廓 和纹理比较模糊, 容易出现块状外观 ${ }^{[4]}$, 产生伪边 缘, 图像质量较差. 插值算法难以处理图像中的模 糊现象、图像引人噪声等问题, 也无法添加先验信 息，因此其适应性较差.
(2) 随着机器学习、深度学习理论的发展, 基 于学习的算法逐渐成为近年较为流行的一类技术. 其基本思想是从训练样本集中学习 LR 图像与 HR 图像之间的映射关系, 从而对未知 LR 图像进行预 测, 达到提高分辨率的目的. 例如, 利用局部嵌人 流行学习的思想的基于邻域嵌人的学习策略 ${ }^{[5]}$, 基 于样例学习的算法 ${ }^{[6-7]}$, 以及基于深度学习的神经 网络算法 ${ }^{[8-10]}$; 但此类算法对外部训练数据集有较 大的依赖性, 模型增量性差. 最新对图像统计数据 的研究表明, 图像块可以由其过完备字典的元素 进行稀疏线性表示, 因此基于稀疏表示 ${ }^{[11-13]}$ 的学 习算法得到应用, 但该算法需要大量的训练数据 集, 且部分数据集需要人工标注, 并且内部字典通 常不足以包含复杂纹理信息而进行良好地重建. 综上, 学习模型对于图像超分辨的效果至关重要, 但目前的模型还无法有效地结合图像重建所需的 全部先验知识, 并且基于学习的算法计算复杂度 较高, 难以满足实时性要求, 因此并不适用于图像 实时处理.

（3）基于重建的算法目的是重建在降质过程 中丢失的高频信号. 假设输人的 LR 图像共有 $n$ 帧, 则基于重建的超分辨问题数学模型可以表示 ${ }^{[14]}$ 为

$$
l_{k}=D B_{k}^{(2)} M_{k} B_{k}^{(1)} H+N_{k}, k=1, \cdots, n
$$

其中, $l_{k}$ 是由待重建的原始 $\mathrm{HR}$ 图像 $H$ 经过一系 列的图像变换过程得到的 LR 图像; $B_{k}^{(1)}$ 是大气模 糊算子; $M_{k}$ 是运动变换算子; $B_{k}^{(2)}$ 是成像模糊算 子; $D$ 是降采样算子; $N_{k}$ 是在成像过程中引人的 加性噪声. 已知输人 $l_{k}$, 则基于重建的算法目标是 寻找真实 $\mathrm{HR}$ 图像 $H$ 的最优估计 $\hat{H}$.

频域法是最早提出的基于重建的超分辨算法 之一, 由 Tsai 等 ${ }^{[15]}$ 于 1984 年提出. 例如, 文献[15] 
分别对 LR 图像和目标 HR 作离散傅里叶变换和连 续傅里叶变换, 并根据傅里叶变换的性质, 在频域 中建立起二者之间的线性关系. Rhee 等 ${ }^{[16]}$ 以离散 余弦变换代替频域的离散傅里叶变换，降低了存 储要求和代价. 虽然频域法的理论简单, 在推导和 计算上都有一定的优势, 但难以添加先验信息及 处理噪声. 另外, 由于频域与空域存在复杂的变换 关系, 只能处理全局整体运动的情况, 难以处理具 有局部运动的情况.

迭代反向投影 (iterative back projection, IBP) 法 ${ }^{[17]}$ 是将退化模型生成的 LR 图像与输人的 LR 图 像二者的差值反向投影到 HR 图像上, 不断迭代使 误差收玫, 从而得到目标 HR 图像. IBP 算法直观, 易于理解, 但 IBP 是逆问题, 它的病态性将导致解 不唯一.凸集投影 (projection onto convex sets, POCS)法是一种采用迭代的 SR 重建算法 ${ }^{[18-19]}$, 其 中可以加人先验信息对结果的影响, 如对目标图 像峰值像素的约束 ${ }^{[18]}$ 等. POCS 算法形式比较灵活, 能够比较方便地添加先验信息, 但算法的计算复 杂度高, 要求多次迭代及投影, 收玫速度比较慢, 算法稳定性不高.

最大后验概率 (maximum a posterior, MAP)算 法是一种基于统计概率的算法框架, 是目前实际 应用和科学研究中运用最多的一类算法. 算法基 本思想源于条件概率, 将已知 LR 图像序列作为观 测结果, 对未知的 HR 图像进行估计. 在 MAP框架 中, 正则项对于控制重建图像的质量起到关键作 用. MAP 模型比较灵活, 尤其在 MAP 框架的正则 项部分, 可以加人对具体问题的具体约束. 因此, 一个有效的正则项是保证 MAP 框架性能的关键. 例如，常用的正则项包括 $L_{2}$ 形式的 Tikhonov 正则 项 ${ }^{[20]}, L_{1}$ 形式的全变差(total variation, TV)正则项 ${ }^{[21]}$
以及双边 TV 正则项 ${ }^{[13]}$, 还有更复杂的 Student- $t$ 正则项 ${ }^{[22]}$. MAP 有完整的理论框架、灵活的空间域 模型以及强大的先验知识包含性. 由此可见, MAP 模型具有较好的适应性、灵活性和鲁棒性, 能够 产生优异的重建结果, 是一种有效的超分辨重建 算法.

基于 MAP 重建算法的种种优势, 本文将 MAP 框架应用于图像超分辨重建技术中, 并结合图像 的自相似性和非局部低秩先验建立一个充分利用 图像蕴含信息的超分辨重建模型. 本文模型以连 续图像序列作为输人, 采用相似块分组技术将单 幅基准图像及前后的图像预处理为高维张量形式, 方便图像块相似性比较, 达到快速块匹配的目的, 提高了计算速度. 在构建的 MAP 模型中加人基于 图像块的非局部低秩先验, 充分利用图像细节, 避 免狭小特征丢失. 通过相似块的奇异值估计待求 块的奇异值, 选取最相似的图像块并进行低秩截 断处理, 从而抑制噪声等微小因素的干扰, 提高重 建图像的质量. 同时, 在每次迭代中交替优化模型 中的参数, 可提高模型的鲁棒性, 避免局部收玫. 最后加权平均重建的图像块, 得出目标 HR 图像.

\section{1 基于 MAP 和非局部低秩模型及求解}

MAP 模型是基于统计概率的算法框架，其基 本思想来源于条件概率, 通过概率最大得出 HR 图 像 $H$ 的最优估计 $\hat{H}$, 进而重建出目标 $\mathrm{HR}$ 图像. 研 究表明, 加人图像相似性 ${ }^{[23-24]}$ 和低秩性 ${ }^{[25]}$ 能有效 地提升重建图像质量. 本节主要介绍针对 LR 图像 序列 $\boldsymbol{Y}\left\{\boldsymbol{y}_{k}\right\}, k=1, \cdots, 10$ 的 MAP 模型, 以及利用自然 图像的非局部自相似性, 采用非局部低秩正则项 对图像重建过程正则化. 模型重建过程如图 1 所示.

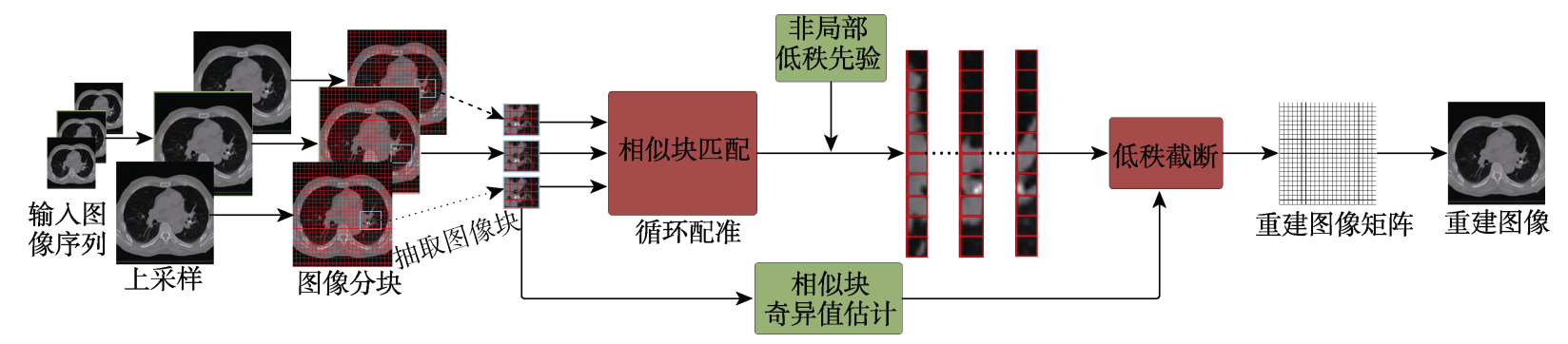

图 1 基于 MAP 和非局部低秩先验的重建模型

\subsection{MAP 模型及求解}

本文中, $\boldsymbol{Y}\left\{\boldsymbol{y}_{k}\right\}$ 由一系列 LR 图像组成, 以序 列中间图像作为基准图像进行超分辨重建, 其他 图像协同辅助. 已知图像退化模型为

$$
\boldsymbol{y}_{k}=\boldsymbol{B}^{(2)} \boldsymbol{D} \boldsymbol{B}_{k}^{(1)} \boldsymbol{x}+\boldsymbol{n}_{k}
$$

其中, $\boldsymbol{x}$ 是待重建的 HR 图像; $\boldsymbol{y}_{k}$ 是输人的 LR 图 像序列, 以序列中间的图像作为基准图像 $\boldsymbol{y} ; \boldsymbol{D}$ 
为下采样算子; $\boldsymbol{B}_{k}^{(1)}$ 为模糊算子; $\boldsymbol{B}^{(2)}$ 为上采样算 子. 假设 $\boldsymbol{n}_{k}$ 是均值为 0 , 方差为 $\sigma_{n}^{2}$ 的加性高斯白 噪声. 超分辨的目的是从 LR 基准图像 $\boldsymbol{y}$ 重建出 $\mathrm{HR}$ 图像 $\boldsymbol{x}$. 该问题可由 MAP 模型表述为

$$
\hat{\boldsymbol{x}}=\arg \max \log P(\boldsymbol{y} \mid \boldsymbol{x}) P(\boldsymbol{x})
$$

研究表明, 自然图像的梯度分布存在重尾现象 ${ }^{[26]}$, 如图 2 所示的城市自然图像(简称 City)的梯度分布 符合重尾分布. 通过对医学断层扫描图像 (CT)梯 度研究, 在医学图像中也存在重尾现象. 因此式 (3)中的第 1 项可以用高斯函数来拟合，即

$$
P(\boldsymbol{y} \mid \boldsymbol{x})=\frac{1}{\sqrt{2 \pi} \sigma_{n}} \mathrm{e}^{\frac{(\boldsymbol{y}-\boldsymbol{x})^{2}}{-2 \sigma_{n}^{2}}}
$$

假设像素值仅与满足吉布斯概率密度函数的 相邻像素有关 ${ }^{[27]}$, 则第 2 项可以用吉布斯函数拟 合，即

$$
P(\boldsymbol{x}) \propto \frac{1}{c} \mathrm{e}^{-\eta J(\boldsymbol{x})}
$$

将式(4)(5)代人式(3), 整理得

$$
\hat{\boldsymbol{x}}=\arg \min \frac{1}{2 \sigma_{n}^{2}}\|\boldsymbol{y}-\boldsymbol{x}\|_{2}^{2}+\eta J(\boldsymbol{x})
$$

即为待求的目标函数, $J(\boldsymbol{x})$ 为正则项.

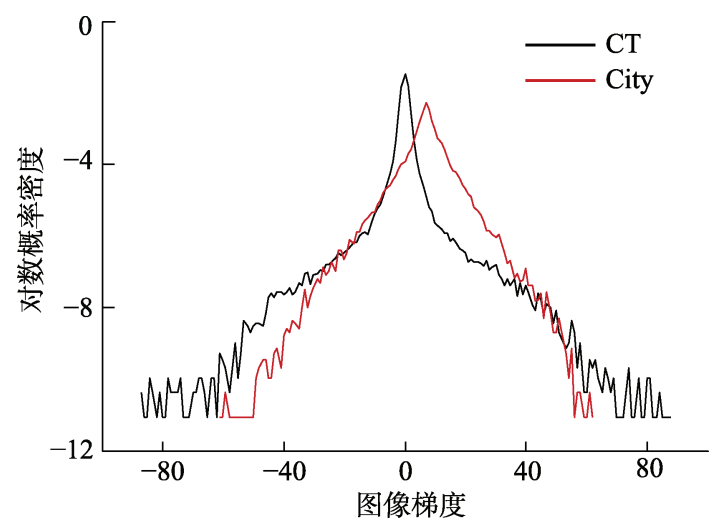

图 2 图像梯度分布

\section{2 非局部低秩正则化及过程}

基于图像块的非局部低秩先验 ${ }^{[28]}$ 在图像去噪 和图像超分辨等方面得到了较好的应用. 本文在上 文工作的基础上加人了非局部低秩先验，并将其表 示为估计. 假设 $\tilde{\boldsymbol{R}}_{j} \boldsymbol{x} \doteq\left[\boldsymbol{R}_{j_{0}} \boldsymbol{x}, \boldsymbol{R}_{j_{1}} \boldsymbol{x}, \cdots, \boldsymbol{R}_{j_{p-1}} \boldsymbol{x}\right] \in \mathbb{R}^{n \times p}$ 表示图像块 $\boldsymbol{x}_{j}$ 的一系列相似块; 其中, $j$ 为图像块 索引, 相似块是以 $j$ 为中心形成的大小为 $\sqrt{n} \times \sqrt{n}$ 的图像块， $\boldsymbol{R}_{j}$ 用于提取图像相似块. 在相似块匹 配时，选取整个序列图像中最相似的 $p$ 块相似块， 再进行低秩截断处理. 假设每组低秩块是相互独
立的，因此低秩先验表示为

$$
\left\{\begin{array}{l}
P(\boldsymbol{x}) \propto \prod_{j} P\left(\tilde{\boldsymbol{R}}_{j} \boldsymbol{x}\right) \\
P\left(\tilde{\boldsymbol{R}}_{j} \boldsymbol{x}\right) \propto \frac{1}{c} \exp \left(-\eta \operatorname{rank}\left(\tilde{\boldsymbol{R}}_{j} \boldsymbol{x}\right)\right)
\end{array}\right.
$$

将式(7)代人式(6), 得

$$
\hat{\boldsymbol{x}}=\arg \min \frac{1}{2 \sigma_{n}^{2}}\|\boldsymbol{y}-\boldsymbol{x}\|_{2}^{2}+\eta \sum_{j} \operatorname{rank}\left(\tilde{\boldsymbol{R}}_{j} \boldsymbol{x}\right)
$$

通常低秩矩阵常用核范式来解决,

$$
\left\{\begin{array}{l}
\hat{\boldsymbol{x}}=\arg \min \frac{1}{2 \sigma_{n}^{2}}\|\boldsymbol{y}-\boldsymbol{x}\|_{2}^{2}+\eta \sum_{j}\left\|\boldsymbol{L}_{j}\right\|_{*} \\
\text { s.t. } \boldsymbol{L}_{j}=\tilde{\boldsymbol{R}}_{j} \boldsymbol{x} .
\end{array}\right.
$$

其中, $\boldsymbol{L}_{j}$ 为待求的 $\tilde{\boldsymbol{R}}_{j} \boldsymbol{x}$ 的低秩块; $\left\|\boldsymbol{L}_{j}\right\|_{*}$ 为 $\boldsymbol{L}_{j}$ 的 核范数, 用以表示奇异值的和. 本文使用迭代方向 乘子, 通过构造增强拉格朗日方程, 求解式(9), 即

$$
\begin{aligned}
& \left(\hat{\boldsymbol{x}}, \boldsymbol{L}_{j}\right)=\arg \min \frac{1}{2 \sigma_{n}^{2}}\|\boldsymbol{y}-\boldsymbol{x}\|+ \\
& \mu \sum_{j}\left\|\tilde{\boldsymbol{R}}_{j} \boldsymbol{x}-\boldsymbol{L}_{j}+\frac{\boldsymbol{U}_{j}}{2 \mu}\right\|_{\mathrm{F}}^{2}+\eta \sum_{j}\left\|\boldsymbol{L}_{j}\right\|_{*}
\end{aligned}
$$

其中, $U_{j}$ 是拉格朗日乘子; $\mu$ 是一个常量参数.

求解式(10)可以分解成 2 个子问题

$$
\left\{\begin{array}{l}
\hat{\boldsymbol{x}}=\underset{\boldsymbol{x}}{\arg \min } \frac{1}{2 \sigma_{n}^{2}}\|\boldsymbol{y}-\boldsymbol{x}\|_{2}^{2}+\mu \sum_{j}\left\|\tilde{\boldsymbol{R}}_{j} \boldsymbol{x}-\boldsymbol{L}_{j}+\frac{\boldsymbol{U}_{j}}{2 \mu}\right\|_{\mathrm{F}}^{2} \\
\boldsymbol{L}_{j}=\underset{\boldsymbol{L}_{j}}{\arg \min } \mu\left\|\tilde{\boldsymbol{R}}_{j} \boldsymbol{x}-\boldsymbol{L}_{j}+\frac{\boldsymbol{U}_{j}}{2 \mu}\right\|_{\mathrm{F}}^{2}+\eta \sum_{j}\left\|\boldsymbol{L}_{j}\right\|_{*}
\end{array}\right.
$$

其中, 对 $\boldsymbol{x}$ 求解，可由式(11)直接求得

$$
\boldsymbol{x}=\left(\boldsymbol{I}+2 \mu \sigma_{n}^{2} \sum_{j} \tilde{\boldsymbol{R}}_{j}^{\mathrm{T}} \tilde{\boldsymbol{R}}_{j}\right)^{-1}\left(\boldsymbol{y}+2 \mu \sigma_{n}^{2} \sum_{j} \tilde{\boldsymbol{R}}_{j}^{\mathrm{T}}\left(\boldsymbol{L}_{j}-\frac{\boldsymbol{U}_{j}}{2 \mu}\right)\right)
$$

其中, $\tilde{\boldsymbol{R}}_{j}^{\mathrm{T}} \boldsymbol{L}_{j}=\sum_{r=0}^{p-1} \boldsymbol{R}_{j_{r}}^{\mathrm{T}} \boldsymbol{x}_{j_{r}} ; \tilde{\boldsymbol{R}}_{j}^{\mathrm{T}} \tilde{\boldsymbol{R}}_{j}=\sum_{r=0}^{p-1} \boldsymbol{R}_{j_{r}}^{\mathrm{T}} \boldsymbol{R}_{j_{r}} ; \boldsymbol{R}_{j_{r}}$ 是在 像素位置 $j_{r}$ 上提取图像块的矩阵. 本文采用 MAP 模型求解低秩块 $\boldsymbol{L}_{j}$, 用 $\tilde{\boldsymbol{R}}_{j} \boldsymbol{x}$ 的奇异值估计出 $\boldsymbol{L}_{j}$ 的 奇异值，从而得出 $\boldsymbol{L}_{j}$.

$$
\hat{\sigma}_{i}\left(\boldsymbol{L}_{j}\right)=\arg \max \log P\left(\sigma_{i}\left(\boldsymbol{L}_{j}\right) \mid \sigma_{i}\left(\tilde{\boldsymbol{R}}_{j} \boldsymbol{x}\right)\right)
$$

其中, $\sigma_{i}\left(\boldsymbol{L}_{j}\right)$ 是 $\boldsymbol{L}_{j}$ 的第 $i$ 个奇异值. 式(13)可由贝 叶斯准则得

$$
\begin{gathered}
P\left(\sigma_{i}\left(\boldsymbol{L}_{j}\right) \mid \sigma_{i}\left(\tilde{\boldsymbol{R}}_{j} \boldsymbol{x}\right)\right)=\frac{P\left(\sigma_{i}\left(\boldsymbol{L}_{j}\right), \sigma_{i}\left(\tilde{\boldsymbol{R}}_{j} \boldsymbol{x}\right)\right)}{P\left(\sigma_{i}\left(\tilde{\boldsymbol{R}}_{j} \boldsymbol{x}\right)\right)} \propto \\
P\left(\sigma_{i}\left(\tilde{\boldsymbol{R}}_{j} \boldsymbol{x}\right) \mid \sigma_{i}\left(\boldsymbol{L}_{j}\right)\right) P\left(\sigma_{i}\left(\boldsymbol{L}_{j}\right)\right)
\end{gathered}
$$

假设扭曲度 $f$ 表示 HR 图像块奇异值与 LR 图像块 奇异值的扭曲度. 其中, 第 1 部分可以看做用均值 
为 0 , 标准差是 $f$ 的高斯函数来拟合，即

$$
\begin{array}{r}
P\left(\sigma_{i}\left(\tilde{\boldsymbol{R}}_{j} \boldsymbol{x}\right) \mid \sigma_{i}\left(\boldsymbol{L}_{j}\right)\right)=\frac{1}{\sqrt{2 \pi} f_{i}} . \\
\quad \exp \left(-\frac{\left(\sigma_{i}\left(\tilde{\boldsymbol{R}}_{j} \boldsymbol{x}\right)-\sigma_{i}\left(\boldsymbol{L}_{j}\right)\right)^{2}}{2 f_{i}^{2}}\right)
\end{array}
$$

另外, $P\left(\sigma_{i}\left(\boldsymbol{L}_{j}\right)\right)$ 可以用核密度估计来计算, 其概 率密度函数被认定为一系列核函数的和, 因此奇 异值的和即为一系列核函数的和, 核函数由以 $\sigma_{i}\left(\boldsymbol{L}_{j}\right)$ 为中心的 $1 \times 3$ 邻域 $\Omega_{i}$ 确定. 本文假设核函 数均值符合均值 $\sigma_{t}\left(\tilde{\boldsymbol{R}}_{j} \boldsymbol{x}\right)$, 标准差为 $h_{i}$ 的高斯分 布，则奇异值的概率密度函数被定义为

$$
P\left(\sigma\left(\boldsymbol{L}_{j}\right)\right) \propto \sum_{\sigma_{i}\left(\tilde{\boldsymbol{R}}_{j} \boldsymbol{x}\right) \in \sigma\left(\tilde{\boldsymbol{R}}_{j} \boldsymbol{x}\right)} \frac{1}{\sqrt{2 \pi} h_{i}} \mathrm{e}^{\left(\frac{\left(\sigma_{i}\left(\boldsymbol{L}_{j}\right)-\sigma_{t}\left(\tilde{\boldsymbol{R}}_{j} x\right)\right)^{2}}{-2 h_{i}^{2}}\right)}
$$

将式(15)(16)中的第 $i$ 个索引代人式(14), 得

$$
\begin{gathered}
P\left(\sigma_{i}\left(\boldsymbol{L}_{j}\right) \mid \sigma_{i}\left(\tilde{\boldsymbol{R}}_{j} \boldsymbol{x}\right)\right) \propto \frac{1}{\sqrt{2 \pi} f_{i}} \\
\exp \left(-\frac{\left(\sigma_{i}\left(\tilde{\boldsymbol{R}}_{j} \boldsymbol{x}\right)-\sigma_{i}\left(\boldsymbol{L}_{j}\right)\right)^{2}}{2 f_{i}^{2}}\right) \times \\
\frac{1}{\sqrt{2 \pi} h_{i}} \exp \left(-\frac{\left(\sigma_{i}\left(\boldsymbol{L}_{j}\right)-\sigma_{t}\left(\tilde{\boldsymbol{R}}_{j} \boldsymbol{x}\right)\right)^{2}}{2 h_{i}^{2}}\right)
\end{gathered}
$$

令式(17)导数为 0 , 解得

$$
\hat{\sigma}_{i}\left(\boldsymbol{L}_{j}\right)=\frac{f_{j}^{2} \sigma_{t}\left(\tilde{\boldsymbol{R}}_{j} \boldsymbol{x}\right)+h_{i}^{2} \sigma_{i}\left(\tilde{\boldsymbol{R}}_{j} \boldsymbol{x}\right)}{f_{j}^{2}+h_{i}^{2}}
$$

再平均所得出的全部 MAP 估计，即

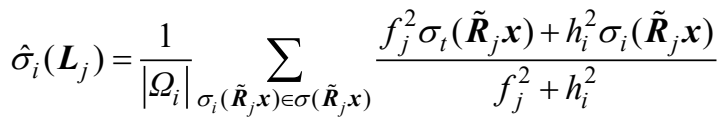

最后得出低秩图像相似块 $\boldsymbol{L}_{j}$ 的估计为

$$
\hat{\boldsymbol{L}}_{j}=\boldsymbol{U}\left(\operatorname{diag}\left(\hat{\sigma}\left(\boldsymbol{L}_{j}\right)\right)\right) \boldsymbol{V}^{\mathrm{T}}
$$

其中, $\boldsymbol{U}\left(\operatorname{diag}\left(\hat{\sigma}\left(\boldsymbol{L}_{j}\right)\right)\right) \boldsymbol{V}^{\mathrm{T}}$ 是 $\tilde{\boldsymbol{R}}_{j} \boldsymbol{x}$ 的奇异值分解形 式; $\hat{\sigma}\left(\boldsymbol{L}_{j}\right)=\sum_{i} \hat{\sigma}_{i}\left(\boldsymbol{L}_{j}\right)$. 增强拉格朗日乘子 $\boldsymbol{U}_{j}$ 更新 式为

$$
\boldsymbol{U}_{j}^{k+1}=\boldsymbol{U}_{j}^{k}+\mu\left(\tilde{\boldsymbol{R}}_{j} \boldsymbol{x}-\boldsymbol{L}_{j}\right)
$$

\section{2 评价指标}

\section{1 峰值信噪比}

峰值信噪比 ${ }^{[29]}$ (peak signal to noise ratio, PSNR) 是一种评价图像与参考图像类似度的客观标准,
衡量经过处理后的图像品质，广泛应用于图像质 量评价. 其计算公式为

$$
\text { PSNR }=10 \times \lg \left(\frac{\left(2^{G}-1\right)^{2}}{\mathrm{MSE}}\right)
$$

其中, MSE 是待评价图像与参考图像之间的均方 误差; $G$ 为图像灰度级数; PSNR 值越大, 待评价图 像与参考图像之间的差异越小, 图像质量越高. 但 PSNR 值只是表示图像质量评价的客观标准，并没 有将人体视觉因素考虑在内, 即便 PSNR 值很高, 但实际图像质量与期望的图像质量还可能存在较 大误差, 所以评价图像质量需要综合分析各种评 价指标.

\section{2 结构相似性}

结构相似性 ${ }^{[30]}$ (structural similarity, SSIM) 是一 种基于退化的图像质量评价方法，通过比较待评 价图像与参考图像之间的结构相似度来判断图像 质量. 在一幅图像中, 每一个像素点对周围像素都 有强依赖性，这些依赖在视觉感知上会携带目标 图像结构的重要信息. 其计算公式为

$$
\operatorname{SSIM}(x, y)=\frac{\left(2 \mu_{x} \mu_{y}+C_{1}\right)\left(2 \sigma_{x} \sigma_{y}+C_{2}\right)}{\left(\mu_{x}^{2}+\mu_{y}^{2}+C_{1}\right)\left(\sigma_{x}^{2}+\sigma_{y}^{2}+C_{2}\right)}
$$

其中, $\mu_{x}$ 和 $\mu_{y}$ 分别是待评价和参考图像的灰度平 均值; $\sigma_{x}$ 和 $\sigma_{y}$ 表示标准差; $C_{1}=\left(k_{1} G\right)^{2}, C_{2}=\left(k_{2} G\right)^{2}$ 作为常量来维持数值稳定性, 其中 $k_{1}=0.01$, $k_{2}=0.03 ; G$ 为图像灰度级数. 事实上, SSIM 是由 与结构信息相关的亮度和对比度来定义的, 平均 灰度值作为亮度测量的估计, 标准差作为对比度 测量的估计. 另外, 因为 SSIM 是对称度量, 所以 可将其视为用于比较任意 2 个信号的相似性度量. 信号可以是离散的或连续的, 并且可以存在任意 维度的空间中。

\section{3 特征相似度}

特征相似度 ${ }^{[31]}$ (feature similarity, FSIM) 是对 SSIM 一种比较成功的变种. 其中, FSIM 中的相位 一致性(phase congruency, PC)用来度量局部结构重 要性, 考虑到 PC 具有对比度不变性, 而对比度又 影响人眼视觉系统对图像质量的感知, 所以在 FSIM 中采用图像梯度幅度 (gradient magnitude, GM)作为 2 级特征. 将 FSIM 划分成 PC 和 GM 2 个部分，其计算公式为

$$
\mathrm{FSIM}=\frac{\sum_{x \in \Omega} S_{L}(x) \cdot \mathrm{PC}_{m}(x)}{\sum_{x \in \Omega} \mathrm{PC}_{m}(x)}
$$


其中, $S_{L}(x)=S_{\mathrm{PC}}(x) \cdot S_{G}(x), S_{\mathrm{PC}}(x)$ 和 $S_{G}(x)$ 分别 是图像间的 $\mathrm{PC}$ 和 $\mathrm{GM}$ 的值; $\mathrm{PC}_{m}(x)$ 是最大的 $\mathrm{PC}$ 值, 用于加权每个点对 2 幅图像整体相似性的贡 献; $x$ 是给定像素点的位置; $\Omega$ 为图像的全部空 域. FSIM 主要以相位相似度和图像梯度相似度来 度量局部结构的重要性, 在评价质量分数阶段, 将 相位相似度作为权值, 增大了与人眼视觉感知的 相关性，取得了良好的质量评估效果.

\section{3 实验结果与分析}

本文的实验图像选用医学图像集、自然图像集 以及视频分帧图像集等各类型图像 5 组, 每组图像 序列 10 幅, 选取其中任意 1 幅图像作为重建图像. 其中, 每幅图像大小是 $128 \times 128$, 重建的 HR 图像 大小是 $256 \times 256$. 实验医学图像数据由山东省千佛 山医院提供. 在测试的自然图像数据集中, 城市数 据集来源于纽约大学理工学院 $\mathrm{Ou}$ 等 $^{(1)}$ 开发的视频 数据库, 该数据库包含使用 H.264 编码的 10 个视 频, 分辨率从 QCIF 到 $4 \mathrm{CIF}$ 不等, 量化参数为 $28 \sim 44$, 帧速率在 3.75 30.00 帧/s, 本文实验只选取 其中的 10 帧作为实验数据. 小果园数据集源于光 流标准实验数据集 ${ }^{2}$, 选取其作为自然图像来测试 本文算法的重建性能. 实验用 Matlab 2014(b)实现 本文图像超分辨重建算法. 实验的硬件设施为 $3.40 \mathrm{GHz}$ Intel(R) Xeon(R) E5-2643 v4 CPU, NVIDIA GeForce GTX 1080M GPU, 256 GB 内存, 操作系统是 Ubuntu 14.04.

实验中使用的图像均是标准连续图像序列, 迭代次数设为 12. 通过 PSNR, SSIM 和 FSIM 评价 实验结果.

\section{1 实验参数}

在本文提出的基于 MAP 和非局部低秩先验的 实验模型中, 通过不同类型对比实验, 优化实验参 数设置. 研究表明, 图像块的大小不仅会影响实验 的运行速度, 还会影响图像配准的精度和重建图像 的质量. 实验分别设置块大小为 $3 \times 3,5 \times 5,7 \times 7,9 \times 9$ 和 $11 \times 11$, 迭代次数均设置为 12 次, 图像块大小对 PSNR, SSIM 和 FSIM 的影响如表 1 和图 3 所示.

由图 3 可见, $3 \times 3,5 \times 5$ 的图像块的数值统计指 标明显高于其余组实验结果. 图像块设置为 $7 \times 7$, $9 \times 9$ 和 $11 \times 11$, 相比于 $5 \times 5$, PSNR 值的数值统计指 标差别较小, 但算法运行效率会随着图像分块大
表 1 图像块大小对 SSIM, FSIM 的影响

\begin{tabular}{ccccccccc}
\hline \multirow{2}{*}{ 图像块 } & \multicolumn{3}{c}{ SSIM } & & \multicolumn{3}{c}{ FSIM } \\
\cline { 2 - 3 } & 最大值 & 平均值 & 最小值 & & 最大值 & 平均值 & 最小值 \\
\hline $3 \times 3$ & 0.9164 & 0.9107 & 0.8989 & & 0.9418 & 0.9416 & 0.9408 \\
$5 \times 5$ & 0.9324 & 0.9321 & 0.9319 & & 0.9398 & 0.9397 & 0.9396 \\
$7 \times 7$ & 0.9312 & 0.9305 & 0.9298 & & 0.9390 & 0.9387 & 0.9384 \\
$9 \times 9$ & 0.9318 & 0.9310 & 0.9301 & & 0.9392 & 0.9389 & 0.9385 \\
$11 \times 11$ & 0.9329 & 0.9322 & 0.9312 & & 0.9396 & 0.9394 & 0.9388 \\
\hline
\end{tabular}

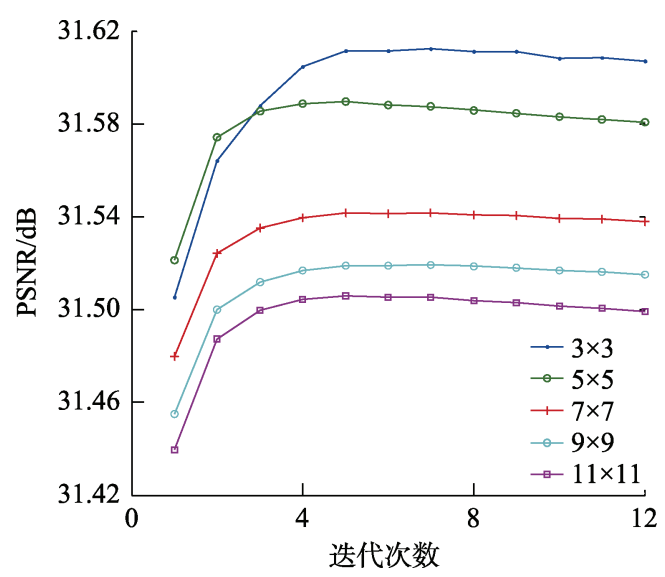

图 3 图像块大小对 PSNR 的影响

小的增加而降低. 结合表 1 的实验结果, $11 \times 11$ 的 图像块仅在 SSIM 中性能较佳; 其中, $3 \times 3$ 与 $5 \times 5$ 的图像块在 FSIM 的 3 项比较中相差最大仅为 0.0020 , 但在 SSIM 比较中, $5 \times 5$ 明显高于 $3 \times 3$, 且 3 项差值为 $0.0330,0.0214$ 和 0.0160 . SSIM 在图像 质量评估中注重局部特征, 在一定范围内, 随着图 像块数增加, 局部特征区域扩大, 图像结构相似度 也越高. 当局部特征区域较小时, 图像结构对比 度、梯度变化较小, FSIM 值较高. 综上所述, 本实 验中图像块大小选取 $5 \times 5$ 为最佳参数设置, 且运行 时间也较 $3 \times 3$ 相差很小, 相对于 $7 \times 7$ 得到较大降 低. 从客观评价指标及下文重建图像视觉效果可 以看出, 对图像进行分块处理能够有效地提升重 建图像的质量. 其中, 实验中正则项迭代次数设置 为 12 次, 迭代次数不同也会对实验结果产生不同 影响. 采用医学肺部图像(CT)和自然图像(City)作 实验数据, 对比不同迭代次数对 PSNR, SSIM 和 FSIM 产生的影响如图 4所示, 其中箭头指向迭代次 数设置为 12 次的数据点. 随着迭代次数的增加, PSNR, FSIM 和 SSIM 值趋于平稳, 但从 PSNR 的曲 线可以看出, 实验结果在 12 次后, 2 组实验在各项 指标上均会有较小的浮动, 相较于 FSIM, SSIM 的 


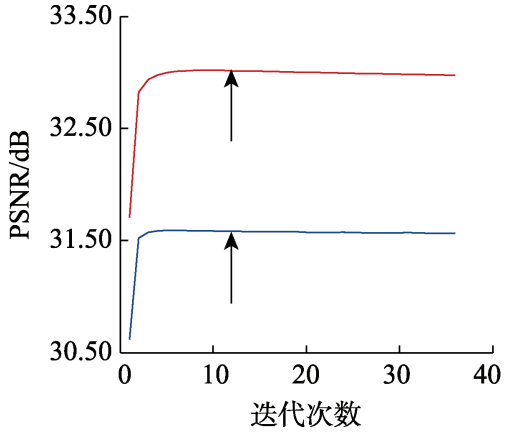

a. PSNR

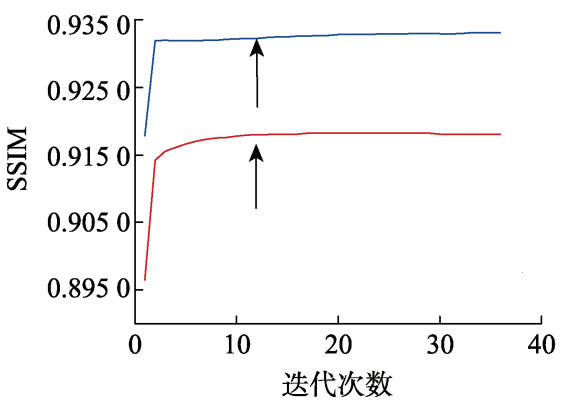

b. SSIM

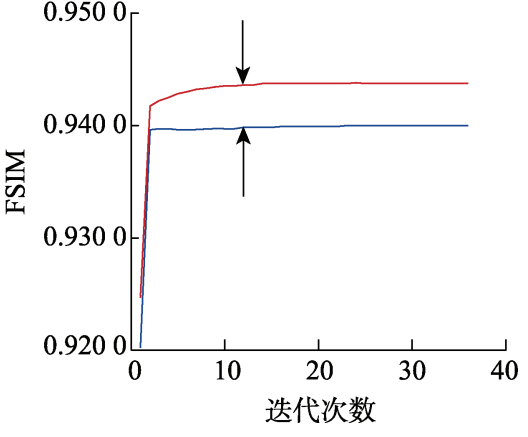

c. FSIM

图 4 迭代次数对 PSNR, SSIM 和 FSIM 的影响

变化曲线相对平缓, PSNR 的曲线有较为明显的下 降趋势; 这是因为基于重建的超分辨率算法中，图 像求解是典型的不适定反问题, 在迭代求解过程中 会引入像噪声等其他微小因素的干扰. 因此, 本文 实验迭代次数设置为 12 次, 避免求解病态问题时 带来的干扰，同时在求解相似块过程中利用低秩截 断能够有效地抑制噪声等微小因素对实验的扰动.

\section{2 对最大后验先验概率项的约束性研究}

不同正则项在基于重建的算法上作用不同， 本文在关于 $L_{2}$ 形式的 Tikhonov 正则项(Tikhonov), $L_{1}$ 形式的 TV 正则项 $\left(L_{1} \mathrm{TV}\right), L_{2}$ 形式的 TV 正则项 $\left(L_{2} \mathrm{TV}\right)$ 、基于低秩与全变分的正则项 ${ }^{[32]}$ (low rank and total variation, LRTV)与本文非局部低秩正则 项(nonlocal low-rank prior, NLR)的研究中, 分别以 迭代次数为 $6,8,10,12$ 和 14 情况下进行测试(为了 简便表达，以迭代次数等于 12 为例).

通过研究 Tikhonov 正则项、 $L_{1} \mathrm{TV}$ 正则项、 $L_{2}$ TV 正则项、LRTV 正则项与 NLR 正则项的对比 实验，使用图像超分辨重建过程中的基准图像进 行测试. 经过实验，本文选取了最具代表性的例 子: 若是 Tikhonov 正则项, 则正则项系数取值为 0.01 ; 若是 $L_{1} \mathrm{TV}$ 正则项，取值为 0.005 ; 若是 $L_{2} \mathrm{TV}$,

则取值为 0.05 ; 若是 LRTV 正则项，均取值为 0.01 ; NLR 正则项, 以 0.1 为测试值. 选取各自最佳实验 结果，进行对比实验，观察与本文正则项的 PSNR, 如表 2 所示.

表 2 迭代次数等于 12 时不同图像的 PSNR 对比 $\mathrm{dB}$

\begin{tabular}{lcc}
\hline 正则项 & CT & City \\
\hline$L_{1} \mathrm{TV}^{[21]}$ & 27.30 & 29.84 \\
$L_{2} \mathrm{TV}^{[21]}$ & 27.86 & 30.67 \\
Tikhonov $^{[20]}$ & 28.19 & 30.96 \\
LRTV $^{[32]}$ & 34.32 & 24.07 \\
NLR & 34.62 & 34.68 \\
\hline
\end{tabular}

由表 2 实验结果可以看出, NLR 与 LRTV 在肺 部图像上的 PSNR 结果要优于 $L_{1} \mathrm{TV}, L_{2} \mathrm{TV}$ 和 Tikhonov 的 PSNR 结果, 并且在城市自然图像上 NLR 的 PSNR 值优于其余 4 项正则项的 PSNR 值, 这说明低秩先验的存在是本文模型效果较好的前 提; 其中, 在 2 幅测试图像的 PSNR 值比较中, NLR 均优于 LRTV, 可以看出非局部低秩先验的存在是 本文模型的核心要素.

图 5 是选择肺部医学图像和城市自然图像作 测试图像产生的实际结果, 虽然总体图像差别不 大，但通过局部展示，可以看出区别更多体现在图
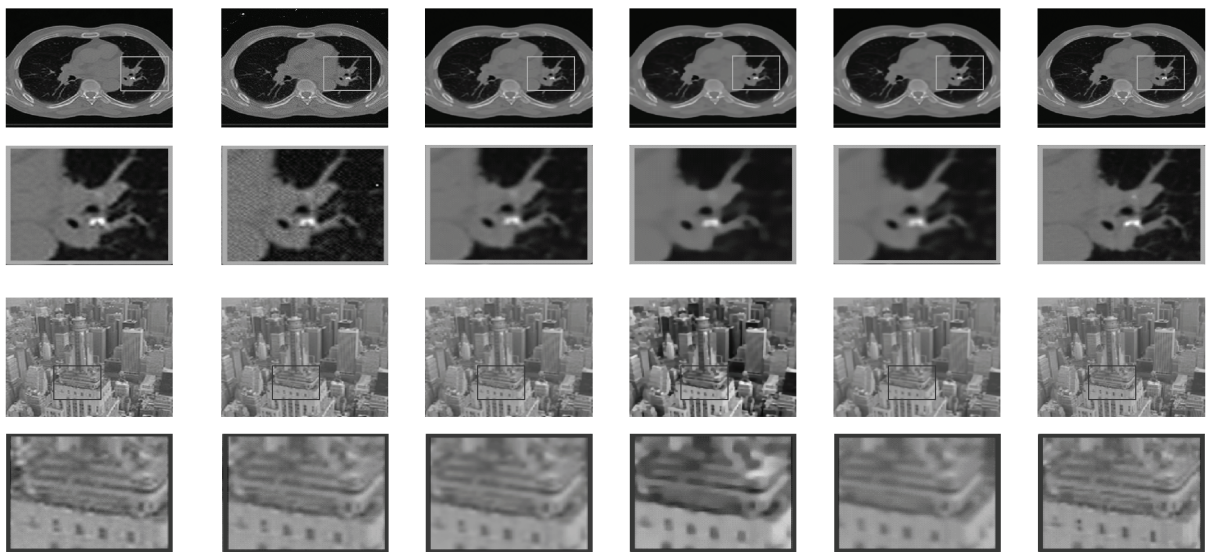

a. $L_{1} \mathrm{TV}^{[21]}$

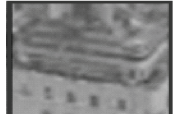

b. $L_{2} \mathrm{TV}^{[21]}$

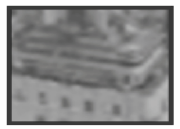

c. Tikhonov ${ }^{[20]}$

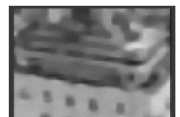

d. LRTV ${ }^{[32]}$

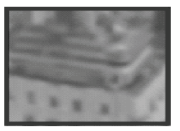

e. NLR

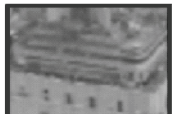

f. 原始图像

图 5 不同正则项的实验对比 
像细节上. 依据共同选取出的图像区域，其中，图 $5 \mathrm{e}$, 图 $5 \mathrm{~d}$ 和图 $5 \mathrm{c}$ 的细节描述能力明显高于图 $5 \mathrm{a}$ 和图 5b. 相对于图 5d 与图 5c, 图 5e 纹理清晰, 没 有产生较重的模糊边缘现象, 这从图像视觉方面 证明了相似块分组存在的必要性以及采取的低秩 截断方法抑制噪声的有效性. 另外, Tikhonov 处理 的图像虽然 PSNR 相对较低, 但其细节还原能力反 而不差, 优于 $L_{1} \mathrm{TV}$ 和 $L_{2} \mathrm{TV}$.

\section{3 实验对比}

本文将模型与 $\mathrm{NNI}^{[3]}$, Bicubic $^{[2]}, \mathrm{LRTV}^{[32]}$ 以及 基于 2-范数二次项解析算法 (a new analytic solution for $\left.L_{2}-L_{2}, L_{2}-L_{2}\right)^{[33]}$ 的图像超分辨处理结果进行 对比. 其中, 在对比实验时, 参数统一, 模型进行
实验所使用的图像均为连续图像序列中的相同图 像, 迭代次数设为 12 .

在本文完整数据集下，本文算法与此 4 种算法 对比计算 PSNR, SSIM 和 FSIM 的结果如表 3 所示, 各图像放大系数均为 $\times 2$.

PSNR 是客观评价图像质量的重要指标之一, 其值的大小可以在很大程度上判定算法超分辨重 建效果的有效性. 而 SSIM 和 FSIM 可以对 PSNR 形成补充参照. 从表 3 可以看出, 在本文使用的图 像数据集下，在对比实验使用的 4 种算法中, $L_{2}-L_{2}$ 的 PSNR 所计算的值相对较高, LRTV 的 SSIM 和 FSIM 所计算的值相对较高, 证明图像的低秩性质 可以很好地重构图像细节.

表 3 不同算法图像超分辨的 PSNR, SSIM 和 FSIM 比较

\begin{tabular}{|c|c|c|c|c|c|c|c|}
\hline \multirow{2}{*}{ 算法 } & \multirow{2}{*}{ 指标 } & \multicolumn{6}{|c|}{ 图像 } \\
\hline & & 肝部 & 肺部 & 建筑物 & 果园 & 花朵 & 全部 \\
\hline \multirow{3}{*}{$\mathrm{NNI}^{[3]}$} & $\mathrm{PSNR} / \mathrm{dB}$ & 19.6900 & 28.4200 & 30.9200 & 18.5800 & 28.6100 & 24.9800 \\
\hline & SSIM & 0.7349 & 0.8785 & 0.8554 & 0.5994 & 0.9504 & 0.8011 \\
\hline & FSIM & 0.8068 & 0.9152 & 0.9138 & 0.8325 & 0.9784 & 0.8893 \\
\hline \multirow{3}{*}{ Bicubic $^{[2]}$} & $\mathrm{PSNR} / \mathrm{dB}$ & 20.8100 & 30.6200 & 33.0300 & 19.7500 & 30.4300 & 26.6600 \\
\hline & SSIM & 0.7664 & 0.9178 & 0.9063 & 0.6395 & 0.9614 & 0.8265 \\
\hline & FSIM & 0.7919 & 0.9202 & 0.9248 & 0.8304 & 0.9779 & 0.8819 \\
\hline \multirow{3}{*}{$\operatorname{LRTV}^{[32]}$} & $\mathrm{PSNR} / \mathrm{dB}$ & 23.0200 & 34.3200 & 22.1000 & 21.7900 & 20.6500 & 24.0600 \\
\hline & SSIM & 0.8157 & 0.9314 & 0.8576 & 0.6800 & 0.9082 & 0.8367 \\
\hline & FSIM & 0.8450 & 0.9343 & 0.9016 & 0.8210 & 0.9603 & 0.8932 \\
\hline \multirow{3}{*}{$L_{2}-L_{2}{ }^{[33]}$} & $\mathrm{PSNR} / \mathrm{dB}$ & 22.1500 & 31.2400 & 32.2400 & 21.1200 & 30.9400 & 27.5600 \\
\hline & SSIM & 0.7133 & 0.8260 & 0.8469 & 0.6374 & 0.8544 & 0.7786 \\
\hline & FSIM & 0.7361 & 0.8692 & 0.8956 & 0.8119 & 0.7482 & 0.8165 \\
\hline \multirow{3}{*}{ 本文 } & $\mathrm{PSNR} / \mathrm{dB}$ & 21.1100 & 34.6200 & 34.3400 & 21.9500 & 31.1100 & 27.3900 \\
\hline & SSIM & 0.7887 & 0.9432 & 0.9256 & 0.7049 & 0.9255 & 0.8497 \\
\hline & FSIM & 0.8223 & 0.9356 & 0.9435 & 0.8443 & 0.9815 & 0.9079 \\
\hline
\end{tabular}

除对比全部数据集的实验结果外，本文还分 别单独列出使用不同算法进行图像超分辨获得的 肺部医学图像、小果园自然图像、城市自然图像, 分别如图 6 图 8 所示. 在图像大小均为 $128 \times 128$ 的肝部图像、肺部图像医学数据集，城市图像、果 园图像和花朵图像自然数据集的 PSNR, SSIM 和 FSIM 的对比结果如表 3 所示. 其中, 全部图像指 不同实验算法对应的 5 组图像数据集的客观评价 指标平均值，不同实验算法对应的实验结果指标 值由上到下依次为 PSNR, SSIM 和 FSIM.

如表 3 所示，对比肝部图像、全部图像，本文 算法的 PSNR 相较于 LRTV 和 $L_{2}-L_{2}$ 差值分别为 $1.91 \mathrm{~dB}$ 和 $0.17 \mathrm{~dB}$, 但在肺部图像、城市图像、小
果园图像和花朵图像中, 本文算法的 PSNR 分别提 升 $0.30 \mathrm{~dB}, 1.31 \mathrm{~dB}, 0.16 \mathrm{~dB}$ 和 $0.17 \mathrm{~dB}$. 在 SSIM 和 FSIM 比较中, 用平均值来衡量 5 组实验数据的 SSIM 和 FSIM 效果, 本文算法的 SSIM 和 FSIM 平 均值分别提升 0.0130 和 0.0147 , 如图 6 中 SSIM 为 0.9432 , 图 7 中 SSIM 为 0.7049 , 图 8 中 SSIM 为 0.9256 . 在对图像进行局部对比时, 本文算法无 论是对于肺部图像的图像重建还是自然图像的超 分辨重建都具有较大的优势. 本文算法能够更好 地保持图像细节，不会产生模糊伪影和锯齿效应. 通过对比重建图像视觉效果和图像质量的指标, 可以看出本文算法均优于其他 4 种算法.

图 6 是从实验数据集选取的一幅肺部图像，在 

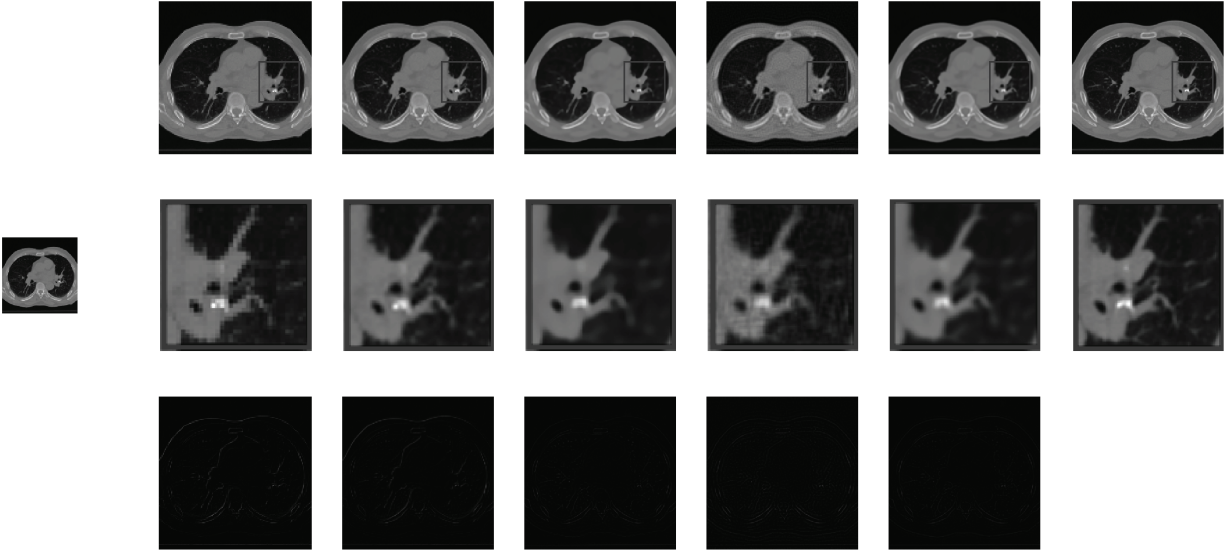

a. 原始图像

b. $\mathrm{NN}^{[3]}$

c. Bicubic $^{[2]}$

d. LRTV ${ }^{[32]}$

e. $L_{2}-L_{2}{ }^{[33]}$

f. 本文

g. Ground Truth

图 65 种算法的医学图像对比
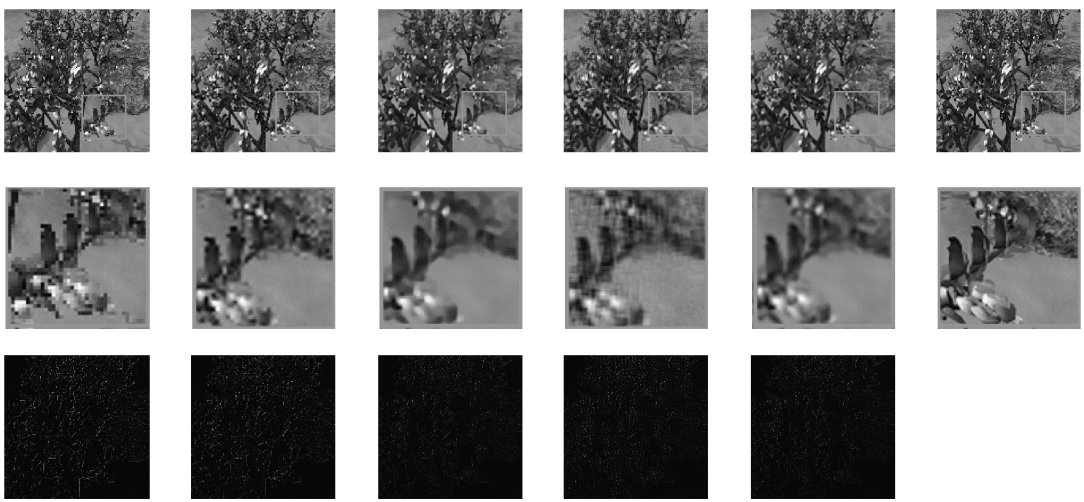

a. 原始图像

b. $\mathrm{NNI}^{[3]}$

c. Bicubic $^{[2]}$

d. LRTV ${ }^{[32]}$

e. $L_{2}-L_{2}{ }^{[33]}$

f. 本文

g. Ground Truth

图 75 种算法的小果园自然图像对比
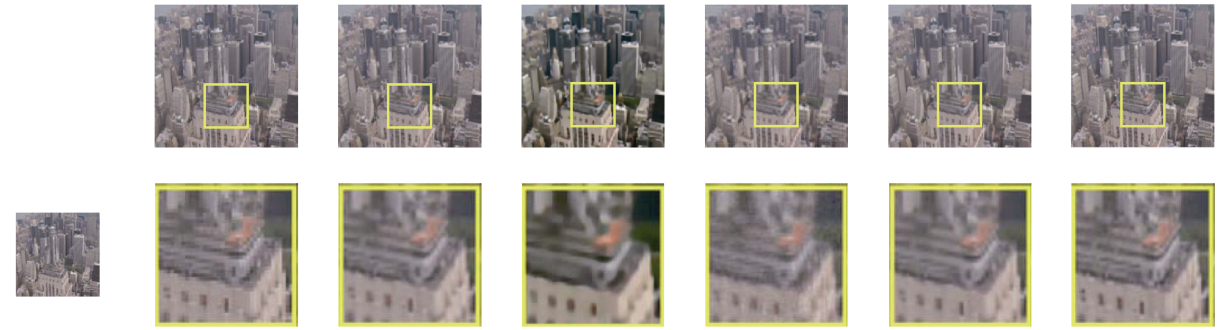

a. 原始图像
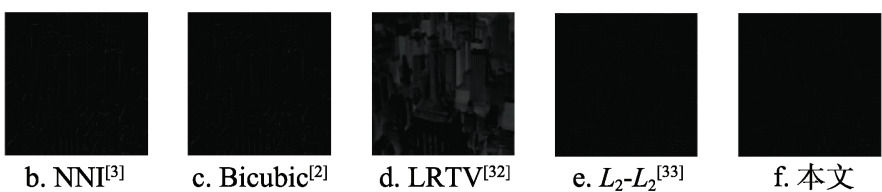

f. 本文

g. Ground Truth

图 85 种算法的城市自然图像对比

5 种重建模型得到的结果图中，原始图像位于最左 侧, Ground Truth 位于图像最右侧, 其余是其中超 分辨算法的排列，验证算法中最后一种算法(位于 Ground Truth 图像左侧)是本文算法. 其中, 将最顶 端的重建图像进行局部展示, 在视觉效果上可以 看出本文算法与 Ground Truth 最为接近. 依据上述 图像可以看出, 图 $6 \mathrm{c}$ 与图 $6 \mathrm{e}$ 最为稳定, 图像波动
不大, 但图 6a 与图 6b 处于一种不稳定态, 尤其图 $6 \mathrm{~b}$ 锯齿效应明显. 图 $6 \mathrm{~d}$ 的局部展示表现了明显的 模糊效应，而在图 $6 \mathrm{e}$ 和图 $6 \mathrm{c}$ 中几乎没有，说明 $L_{2}-L_{2}$ 无法有效地保留图像细节. LRTV 与本文算法 最为接近, 但容易模糊图像纹理, 造成图像边缘模 糊. 从最底端的误差结果图也可以看出, 本文算法 与原图差异较小, 重建图像质量高的同时极其稳定. 
自然图像包含大量的细节信息，有效地重建 出图像, 高频信息对图像质量尤为重要. 从图 7 和 图 8 可以看出，图 7a、图 $8 \mathrm{a}$ 和图 $7 \mathrm{~b}$ 、图 $8 \mathrm{~b}$ 的高 频信息无法有效地还原，进而影响重建图像质量， 造成边缘纹理模糊和块状伪影. 观察自然图像的 局部展示，可以看出图 $7 \mathrm{c}$ 和图 $8 \mathrm{c}$ 会出现对边缘纹 理粗化的状况，说明在实现细节保留上，低秩正则 化与全变分正则化有叠加效应，造成图像细节无 法有效地还原. 图 $7 \mathrm{~d}$ 和图 $8 \mathrm{~d}$ 在图像高频细节部分 产生颗粒状伪影，不能有效地保存平滑区域的图 像信息，从而造成在纹理边缘处产生模糊效应，不 能抑制噪声干扰，重建图像清晰度较差. 相比以上 4 种算法, 本文算法可以更好地保留图像细节, 在 图像平滑区域的重建过程中能够有效地抑制噪声 等微小因素的干扰，进而提升重建结果图像质量.

\section{4 结 语}

本文对 MAP 框架进行了改进，使用非局部低 秩先验作为正则化项，提出一种结合非局部低秩 先验和最大后验框架的模型进行图像超分辨重建. 首先，本文模型采用连续图像序列作为数据输人， 利用图像的非局部自相似性作为先验知识, 结合 图像块局部分组技术将相似块进行块匹配，充分 挖掘图像像素级的空间结构关系. 其次, 在本文的 MAP 框架中，通过相似块奇异值的最大后验估计 出待求块的奇异值, 采用低秩截断的方法抑制噪 声等微小因素的干扰. 最后, 采用非局部低秩约束 正则化图像重建过程, 充分利用单幅图像内、连续 图像间的局部和全局信息，提升目标 HR 图像质 量. 本文模型采用多个评价指标：PSNR，SSIM 等， 并表现出优势. 实验结果表明, 本文超分辨模型在 图像超分辨重建上具有良好的鲁棒性. 但是，在低 秩截断过程中，一些狭小细节被丢失了. 研究发 现，残差在保持细节方面具有很好的效果，如何将 残差加人模型，进一步保持图像细节是下一步工 作重点.

\section{参考文献(References):}

[1] Tong C S, Leung K T. Super-resolution reconstruction based on linear interpolation of wavelet coefficients[J]. Multidimensional Systems and Signal Processing, 2007, 18(2/3): 153-171

[2] Carey W K, Chuang D B, Hemami S S. Regularity-preserving image interpolation[J]. IEEE Transactions on Image Processing, 1999, 8(9): 1293-1297
[3] Olivier R, Cao H Q. Nearest neighbor value interpolation[J]. International Journal of Advanced Computer Science and Applications, 2012, 3(4): 25-30

[4] Tian J, Ma K K. A survey on super-resolution imaging[J]. Signal Image and Video Processing, 2011, 5(3): 329-342

[5] Chang H, Yeung D Y, Xiong Y M. Super-resolution through neighbor embedding[C] //Proceedings of the IEEE Computer Society Conference on Computer Vision and Pattern Recognition. Los Alamitos: IEEE Computer Society Press, 2004, 1: 275-282

[6] Datsenko D, Elad M. Example-based single document image super-resolution: a global MAP approach with outlier rejection[J]. Multidimensional Systems and Signal Processing, 2007, 18(2): 103-121

[7] Glasner D, Bagon S, Irani M. Super-resolution from a single image[C] //Proceedings of the 12th IEEE International Conference on Computer Vision. Los Alamitos: IEEE Computer Society Press, 2009, 1: 349-356

[8] Xu Jun, Liu Hui, Guo Qiang, et al. Super-resolution reconstruction of $\mathrm{CT}$ images using neural network combined with deconvolution[J]. Journal of Computer-Aided Design \& Computer Graphics, 2018, 30(11): 2084-2092(in Chinese) (徐军, 刘慧, 郭强, 等. 结合反卷积的 $\mathrm{CT}$ 图像超分辨重 建网络 $[\mathrm{J}]$. 计算机辅助设计与图形学学报, 2018, 30(11): 2084-2092)

[9] Huang H J, Yang J, Huang $\mathrm{H}$, et al. Deep learning for super-resolution channel estimation and DOA estimation based massive MIMO system[J]. IEEE Transactions on Vehicular Technology, 2018, 67(9): 8549-8560

[10] Li S M, Fan R, Lei G Q, et al. A two-channel convolutional neural network for image super-resolution[J]. Neurocomputing, 2018, 275(31): 267-277

[11] Yang J C, Wright J, Huang T S, et al. Image super-resolution via sparse representation[J]. IEEE Transactions on Image Processing, 2010, 19(11): 2861-2873

[12] Wang J J, Zhu S H, Gong Y H. Resolution enhancement based on learning the sparse association of image patches[J]. Pattern Recognition Letters, 2010, 31(1): 1-10

[13] Kim K I, Kwon Y. Single-image super-resolution using sparse regression and natural image prior[J]. IEEE Transactions on Pattern Analysis and Machine Intelligence, 2010, 32(6): 1127-1133

[14] Farsiu S, Robinson M D, Elad M, et al. Fast and robust multiframe super resolution[J]. IEEE Transactions on Image Processing, 2004, 13(10): 1327-1344

[15] Tsai R Y, Huang T S. Multi-frame image restoration and registration $[\mathrm{J}]$. Advances in Computer Vision and Image Processing, 1984, 1(2): 317-339

[16] Rhee S, Kang M G. Discrete cosine transform based regularized high-resolution image reconstruction algorithm[J]. SPIE Journal Optical Engineering, 1999, 38(8): 1348-1356

[17] Irani M, Peleg S. Improving resolution by image registration[J]. CVGIP:Graphical Models and Image Processing, 1991, 53(3): 231-239

[18] Stark H, Oskoui P. High-resolution image recovery from image-plane arrays, using convex projections[J]. Journal of the Optical Society of America. A, Optics and Image Science, 1989, 6(11): 1715-1726 
[19] Banham M R, Katsaggelos A K. Digital image restoration[J]. IEEE Signal Processing Magazine, 1997, 14(2): 24-41

[20] Hennings-Yeomans P H, Baker S, Kumar B V K V. Simultaneous super-resolution and feature extraction for recognition of low-resolution faces[C] //Proceedings of the IEEE Conference on Computer Vision and Pattern Recognition. Los Alamitos: IEEE Computer Society Press, 2008: 1-8

[21] Babacan S D, Molina R, Katsaggelos A K. Total variation super resolution using a variational approach[C] //Proceedings of the 15th IEEE International Conference on Image Processing. Los Alamitos: IEEE Computer Society Press, 2008: 641-644

[22] Chantas G, Galatsanos N, Likas A, et al. Variational Bayesian image restoration based on a product of t-distributions image prior[J]. IEEE Transactions on Image Processing, 2008, 17(10): 1795-1805

[23] Li Y H, Song B, Guo J, et al. Super-resolution of brain MRI images using overcomplete dictionaries and nonlocal similarity[J]. IEEE Access, 2019, 7(1): 25897-25907

[24] Zhan Yuli, Chi Jing, Ye Yanan, et al. Super resolution image reconstruction based on image similarity and feature combination[J]. Journal of Computer-Aided Design \& Computer Graphics, 2019, 31(6): 1018-1029(in Chinese) (战玉丽, 迟静，叶亚男，等. 基于图像相似性和特征组合的 超分辨图像重建 $[\mathrm{J}]$. 计算机辅助设计与图形学学报, 2019, 31(6): 1018-1029)

[25] Huang Detian, Huang Weiqin, Yun Haijiao, et al. Application of regularization technique and low-rank matrix in super-resolution algorithm via sparse representation[J]. Journal of Computer-Aided Design \& Computer Graphics, 2018, 30(5): 868-877(in Chinese)
(黄德天，黄炜钦，云海姣，等. 正则化技术和低秩矩阵在稀 疏表示超分辨率算法中的应用 [J]. 计算机辅助设计与图形 学学报, 2018, 30(5): 868-877)

[26] Krishnan D, Fergus R. Fast image deconvolution using hyper-Laplacian priors[C] //Proceedings of the 22nd International Conference on Neural Information Processing Systems. Cambrige: MIT Press, 2009: 1033-1041

[27] Chen J, Nunez-Yanez J, Achim A. Video super-resolution using generalized Gaussian Markov random fields[J]. IEEE Signal Processing Letters, 2012, 19(2): 63-66

[28] Huang T, Dong W S, Xie X M, et al. Mixed noise removal via laplacian scale mixture modeling and nonlocal low-rank approximation[J]. IEEE Transactions on Image Processing, 2017, 26(7): 3171-3186

[29] Welstead S. Fractal and wavelet image compression techniques[M]. Bellingham: Society of Photo-Optical Instrumentation Engineers, 1999

[30] Wang Z, Bovik A C, Sheikh H R, et al. Image quality assessment: from error visibility to structural similarity[J]. IEEE Transactions on Image Processing, 2004, 13(4): 600-612

[31] Zhang L, Zhang L, Mou X Q, et al. FSIM: a feature similarity index for image quality assessment[J]. IEEE Transactions on Image Processing, 2011, 20(8): 2378-2386

[32] Shi F, Cheng J, Wang L, et al. LRTV: MR image super-resolution with low-rank and total variation regularizations[J]. IEEE Transactions on Medical Imaging, 2015, 34(12): 2459-2466

[33] Zhao N N, Wei Q, Basarab A, et al. Fast single image superresolution using a new analytical solution for $L_{2}-L_{2}$ problems[J]. IEEE Transactions on Image Processing, 2016, 25(8): 3683-3697 